\title{
Evaluating the Performance of Geographic Features Detection Approaches in Urban Areas Based on Unmanned Aerial Vehicle (UAV) Images ${ }^{(*)}$
}

\author{
Omar Aldayafleh \\ Department of Geography, \\ Yarmouk University, Irbid, \\ Jordan
}

\author{
Ahmad khawaldeh \\ Department of Geography, \\ Yarmouk University, Irbid, \\ Jordan
}

\author{
Ali Almagbile \\ Department of Geography, \\ Yarmouk University, Irbid, \\ Jordan
}

\section{Abstract}

Many detection techniques have already been used in extracting geographic information from UAV images to perform various photogrammetric and mapping activities. Among these techniques is the Feature from Accelerated Segment Test (FAST) and the Harris corner detector. It is widely agreed that the evaluation of detectors is of great importance because it evaluates and enhances the accuracy of the detected features. This research evaluates the performance of FAST-9 and FAST-12 as well as the Harris detector in terms of the repeatability rate, completeness, and correctness under different threshold values. Each method is evaluated in terms of its ability for detection unmanned aerial vehicle (UAV) objects (crowd and cars features). Then the common detected features between both FAST versions and the Harris detector are extracted. This is to determine which method performs best under different image conditions (e.g., illumination variations, camera position and orientation, and image noise). The results show that the size of the threshold plays a crucial role in determining the number of detected feature points. An increase in the threshold value leads to a decrease in the number of detected points and vice versa. Thus, the correctness decreases whereas the completeness increases as a function of the threshold values. Furthermore, the relationship between the FAST-9 and the Harris detector is slightly better than those between the FAST-12 and the Harris detector. This is because the number of common

${ }^{(*)}$ Bulletin of the Faculty of Arts Volume 80 Issue 5 July 2020 
features between the FAST-9 and the Harris detector are relatively higher than those between the FAST-12 and the Harris detector.

Keywords - OBIA, Features detection, FAST algorithm, Harris detector, UAV

تقييم أداء طرق استخلاص الظواهر الجغر افية في المناطق الحضرية اعتمادا على صور الطائر ات بلدون طيار

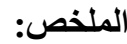

تتعدد التقنيات المستخدمة في استخلاص المعلومات الجغرافية من الطائرات المسيرة

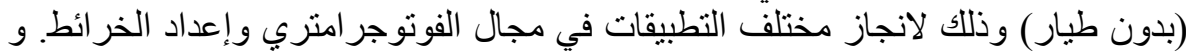

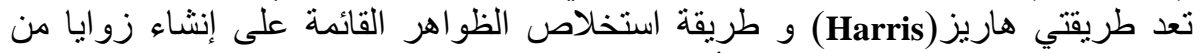

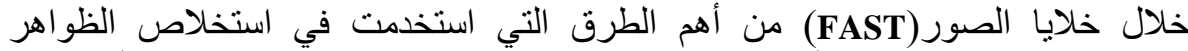

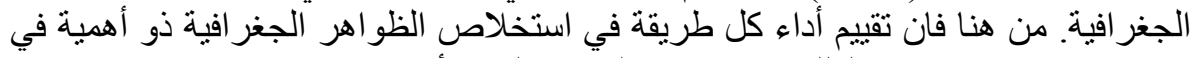
تحسين دقة الاستخلاص. لذللك جاءت هذه الدراسة لتهنة لتقييم أداء

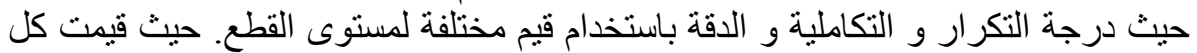

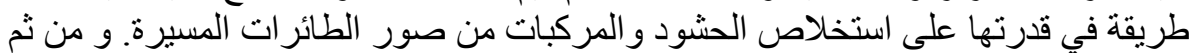

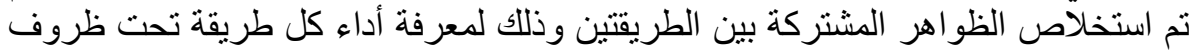

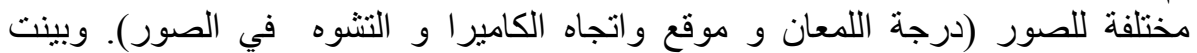

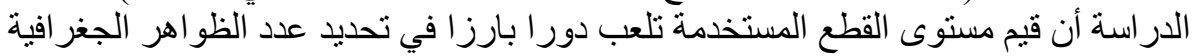

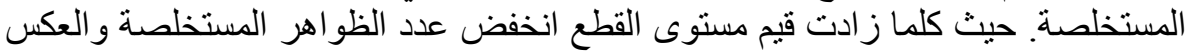

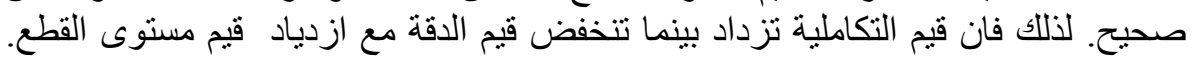

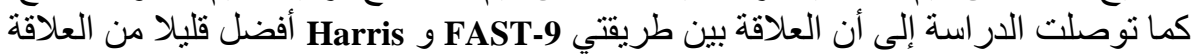

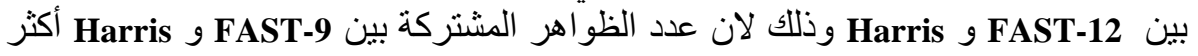

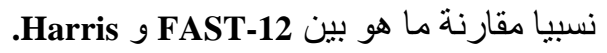
الكلمات الدالة: استخلاص الظواهر من خلال تحليل الصور ، استخلاص الظواهر الجغر افية، طريقة FAST، طريقة الطية Harris 


\section{INTRODUCTION}

In recent years, considerable attention has been directed towards feature extraction from unmanned aerial vehicles (UAVs) images by using either pixel-based or object-based image analysis (OBIA). In pixel-based image analysis, image information is extracted from a single pixel based on the intensity of the pixel value [1]. Widely known corner detection algorithms include the Smallest Univalue Segment Assimilating Nucleus (SUSAN), the Feature from Accelerated Segment Test (FAST), and the Harris corner detector. The OBIA method [2-5] extracts image information based on a number of homogeneous pixels that form meaningful geographic objects. Both pixel and object based image analysis have been intensively studied in many research disciplines such as civil engineering, photogrammetry, and social sciences [6-7] to perform specific detection and mapping tasks.

Crowd feature extraction for monitoring and management is one of the tasks that is based on a pixel-based image analysis (e.g., corner detection algorithms). The importance of using corner detection algorithms for crowd feature detection, analyzing, and managing has particularly taken place in popular events (e.g., sports, concerts) to develop safety strategies at national levels, avoiding crowd related disasters, and enhancing public safety. As a result, many research projects have already developed corner detection approaches for crowd analysis such as detecting, counting, and estimating crowd density. Examples of these projects funded by Europe Union (EU) are PRISMATICA, ADVISOR, SERKET projects [6].

In any crowd research approach, crowd information needs to be extracted to design a model for crowd management and monitoring particularly in overcrowded areas. This requires designing a framework for which includes sensing, alerting, and action stages for crowd management applications [7]. The Sensing step includes capturing the crowd scene using either images or video recordings from land or air-based platforms. The alerting step processes crowd images or videos data using different image processing techniques for crowd feature detection and selection. The extracted information is then used for the action stage to ensuring safety movements and finding alternative pathways for people.

UAVs are one of the maneuvering platforms which have been exploited 
for capturing crowd images and videos. UAVs offer a low cost platform, offer flying flexibility, can fly at low altitudes, and are fast and light weight [8-10] in which makes it ideal for performing crowd monitoring activities. Many studies [11-13; 7] have already utilized the UAVs images for detecting, counting, and estimating crowd density.

Crowd features detection and density estimation have been achieved using either pixels or texture statistics approaches. Marana et al. [14] proposed a method based on Grey Level Dependency Matrix (GLDM) to estimate crowd density. This method assumes that the coarse texture presents a low crowd density whereas a high crowd density is presented by fine texture. Davies et al. [15] developed a background removal technique to estimate crowd density based on pixel statistics, while Yin et al. [16] used a reference image with only a background to classify image pixels into background or foreground (crowd features). Similarly, Ma et al. [17] employed background removal technique to calculate and estimate crowd density, and Kong et al. [18] employed background subtraction and edge detection to detect crowd features. Another technique, based on the information fusion [19], has been employed for estimating the number of people from a group of image sensors. Corner detection procedures, such as the Features from Accelerated Segment Test (FAST) and the Harris corner detector, have been utilized to detect and estimate crowd density from airborne images. Xu et al. [20] proposed an improved Harris corner detection method to detect crowd features, and then performed a clustering analysis from a coordinate matrix of those feature corners. Other researchers [e.g., 21-22; 7] used the FAST algorithm for crowd feature extraction and density estimation. For instance, a new testing procedure based on FAST method (FAST-9 and FAST-12) was proposed by Almagbile [7] for mapping the levels of crowd density.

This research evaluated the performance of the FAST-9, FAST-12, and the Harris detector in detecting UAV image objects (crowd and cars features). A comparison between those algorithms was conducted to select the best method which provided an accurately estimate of the crowd and car features. The performance of each method was tested based on the correctness and completeness criteria. 


\section{REVISITING OF LOCAL FEATURE DETECTORS} AND DESCRIPTORS

\subsection{FAST}

Feature from the Accelerated Segment Test (FAST) method is originally proposed by Rosten and Drummond [23] and is used for identifying the corner points or interest points, from the intensity of pixel values. Similar to the Smallest Uni-value Segment Assimilating Nucleus Test (SUSAN) detector, the FAST algorithm uses a Bresenham's circle of diameter 3.4 pixels as a test mask. This mask consists of 16 pixels around a center pixel Ip. This center pixel can be a corner if at least a number of connected pixels are brighter or darker than the threshold determined by the center pixel Ip [24; 7].

The FAST algorithm determined the centre pixel Ip as a corner based on the intensity values of its neighbourhood pixels. Mathematically, it can be written as $[25 ; 26]$

$S_{p \rightarrow x}=\left\{\begin{array}{ccc}d, & I_{p \rightarrow x} \leq I_{p}-t & \text { darker } \\ s, & I_{p}-t<I_{p \rightarrow x}<I_{p}+t & \text { similar } \\ b, & I_{p \rightarrow x} \geq I_{p}+t & \text { brighter }\end{array}\right.$

Where $S_{p \rightarrow x}$ is the state of pixels around the center pixel Ip, $t$ denotes threshold, $\mathrm{Pd}$ are darker pixels than the intensity of center pixel $\mathrm{Ip}-\mathrm{t}, \mathrm{Pb}$ are brighter pixels than the intensity of center pixel Ip+t and Ps has similar pixels to the intensity of the center pixel Ip.

In order to speed the test up, four pixels, namely I1, I5, I9, and I13, are firstly compared with the center pixel Ip. If those pixels are darker or brighter than Ip, other pixels are tested to check whether 9 (FAST-9) or 12 (FAST-12) connected pixels are higher or lower than the value of the Ip. When the four pixels do not pass the test, early rejection of the Ip as a possible corner point can be achieved.

Different FAST versions (e.g., FAST-9, FAST-12, and FASTER) have been developed to improve the quality of the test in terms of repeatability and the speed of the test. Among these FAST versions is the FAST-9. It has been determined that FAST-9 has the best segment test results because it 
performs the highest repeatability with high test speed [25]

\subsection{HARRIS}

One of the most popular detector methods which have been used for corner detection is the Harris method [27]. This method is an extension of Moravec's corner detector which is based on the detection of a moving window in an image and determines the average changes of image intensity that result from the shifting a window by a small amount in various directions [27]. Assuming the image intensities I and the change $\mathrm{E}(\mathrm{x}, \mathrm{y})$ produced by a shift $(\mathrm{x}, \mathrm{y})$ is written as:

$$
E_{(x, y)}=\sum_{(u, v)} w_{u, v}\left|I_{x+u, y+v}-I_{u, v}\right|^{2}=[x, y] M(x, y)^{T}
$$

Where $w_{u, v}$ is a window function, the symmetric matrix $\mathrm{M}$ is written as:

$$
M=\left[\begin{array}{ll}
A_{(x, y)} & C_{(x, y)} \\
C_{(x, y)} & B_{(x, y)}
\end{array}\right]
$$

Where:

$$
\begin{aligned}
& A_{(x, y)}=I X^{2} \otimes G_{(x, y)} \\
& B_{(x, y)}=I Y^{2} \otimes G_{(x, y)} \\
& C_{(x, y)}=I X Y \otimes G_{(x, y)}
\end{aligned}
$$

$\mathrm{E}$ is related to the local autocorrelation function. Let $\alpha$ and $\beta$ denoting the eigenvalues of $\mathrm{M}, \mathrm{E}(\mathrm{x}, \mathrm{y})$ increases in all shift if both $\alpha$ and $\beta$ are large. The trace $M=\alpha+\beta$ and the Det $M=\alpha, \beta$. The extraction of local corners, or interest points, from Harris corner detection $\mathrm{R}$ is based on a thresholding and can be written as:

$$
R=\operatorname{Drt}(M)-k(\operatorname{Trace}(M))^{2}
$$

\section{METHODOLOGY}

The performances of these detectors were evaluated by Heipke et al (1997), Rottensteiner et al (2007) and Almagbile (2019). They all used completeness, correctness, and a repeatability analysis under different 
imaging conditions in terms of image rotations, scales, and illumination variations. The completeness and correctness can be respectively written as [28-29; 7]:

$$
\begin{aligned}
& \text { completeness }=\frac{T P}{T P+F N} \\
& \text { correctness }=\frac{T P}{T P+F P}
\end{aligned}
$$

Where TP (the number of true positives) is the number of entities found to be available in both reference and experimental images, FN (the number of false negatives) denotes the number of entities in the reference dataset that are not detected automatically. FP (the number of false positives) is the number of entities that are detected in the experimental images but do not correspond to an entity in the reference dataset.

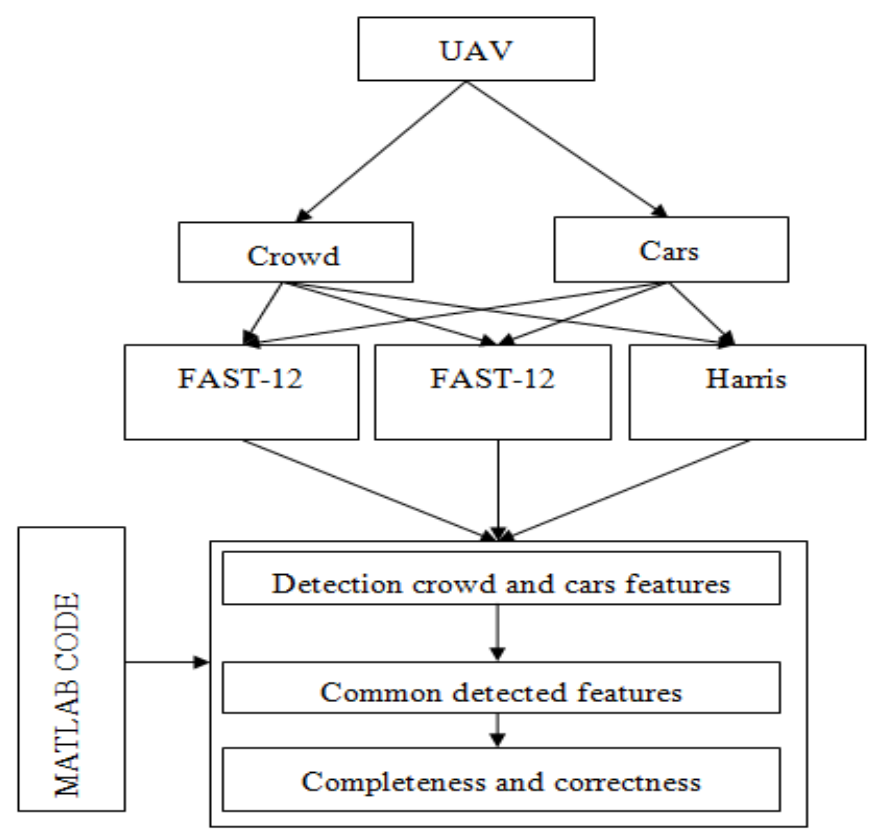

Figure 1. Flow chart of the methodology 
The repeatability compares the geometrical stability of the detected features between different images of a given scene taken under different imaging conditions (noise, camera position-orientation, and lighting variations). Thus, reference point features (ground truth) are detected by a corner detection method (e.g., FAST). The accuracy is high when the detected features in reference detected points are repeated in by the experimental corner detection method (e.g., Harris detector) [30]. The repeatability rate is the percentage of the total corner points (interest points) that are detected in two corner detection algorithms (the reference and the experimental methods) with the same image. This can be obtained through dividing the number of redetected points in the experimental method by the number of points in the reference method [31]:

$$
D_{r}=\frac{n_{\text {method }}}{\sum n_{\text {ref }}}
$$

Where $D r$ denotes the repeatability rate (re-detection rate), $n_{\text {exp }}$ is the number of the re-detected points in the experimental method, $\sum n_{\text {ref }}$ denotes the total number of the detected points in the reference method. Therefore, the best detection method should be reliable and ideally deliver the same points under all possible imaging conditions.

The flow chart of the research methodology is shown in Figure 1.

\subsection{STUDY AREA AND RESEARCH DATA}

Two UAV images (Figure 2) are used in this research to test the performance of the FAST (FAST-9, FAST-12) and the Harris corner detectors for detection UAV image objects (crowd and cars features). The first image, which included the crowd, was extracted from online video (www.greenpeace.org/usa/aerial-video-immense-crowd-peoplesclimate-march)

The second UAV image shows cars in a parking lot and was collected from an in-situ UAV flight for this study. The UAV flight mission was conducted in the spring of 2019 (12 April 2019) over the Aqaba City, Jordan. This UAV was deployed with sensors (GPS/IMU/digital camera) to 
map the geographic features over the area under consideration. It also includes several functions such as auto-pilot operation, no-fly zones, and auto-return home. The specifications of this UAV system are provided by the manufacturer and are shown in Table 1.
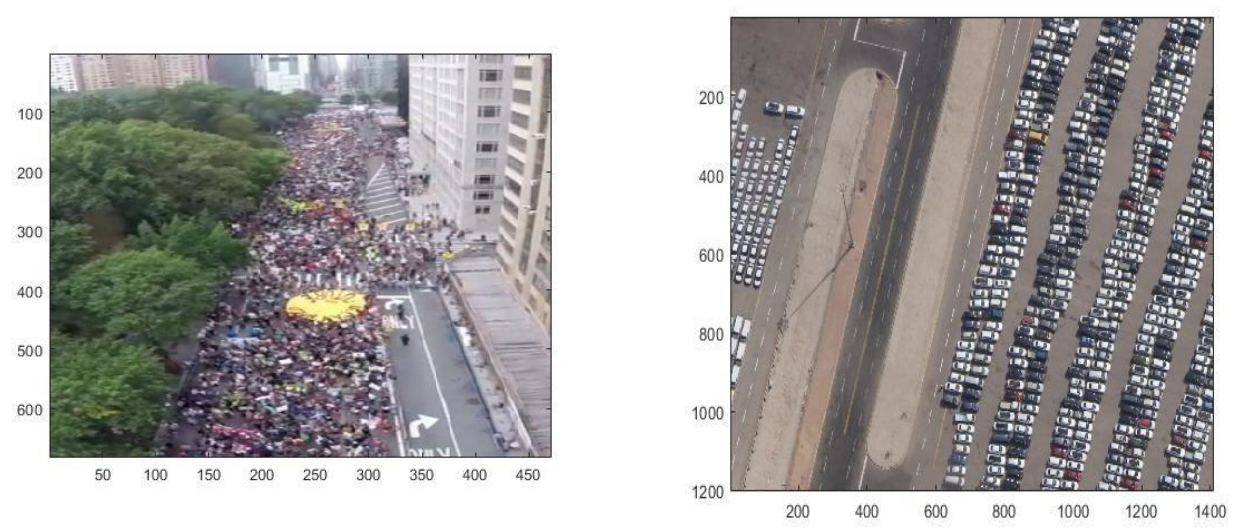

A

B

Figure 2. Experimental images: crowd features extracted from online video

(A) and parking lot image from a real UAV flight (B).

Table 1. Specifications of the UAV used in this research.

\begin{tabular}{|l|l|}
\hline \multicolumn{1}{|c|}{ UAV Features } & \multicolumn{1}{c|}{ Feature Specifications } \\
\hline Weight (Battery and Propellers & $1030 \mathrm{~g}$ \\
Included) & Vertical: $\pm 0.8 \mathrm{~m}$, Horizontal: \pm 2.5 \\
(Stationary) Hover Accuracy & $\mathrm{m}$ \\
Max Yaw Angular Velocity & $200^{\circ} / \mathrm{s}$ \\
Max Tilt Angle & $35^{\circ}$ \\
Max Ascent/Descent Speed & Ascent: $6 \mathrm{~m} / \mathrm{s} ;$ Descent: $2 \mathrm{~m} / \mathrm{s}$ \\
Max Flight Speed & $15 \mathrm{~m} / \mathrm{s}($ Not Recommended) \\
Diagonal Length & $350 \mathrm{~mm}$ \\
Power Consumption & $5.6 \mathrm{~W}$ \\
Flight Time & $25 \mathrm{minutes}$ \\
Take-Off Weight & $\leq 1300 \mathrm{~g}$ \\
Operating Temperature & $-10^{\circ} \mathrm{C} \sim 50^{\circ} \mathrm{C}$ \\
\hline
\end{tabular}




\section{Results and Discussion}

\subsection{Crowd features extraction and quantitative evaluation}

Figures 3 and 4 show the extracted features from a horizontal UAV image using the FAST-9, the FAST-12 and Harris detectors. The common detected features between the Harris and both the FAST-9 and FAST-12 are also attached to these Figures. It can be seen that the size of the threshold plays a crucial role in determining the number of detected features. This is noticeable when comparing the number of detected features by the FAST-9 with different threshold values. The FAST -9 with a threshold of \pm 40 , detects more crowd features when compared with the FAST-12 with a threshold of \pm 60 . A similar situation is noticeable in the case of the Harris detector with threshold values of 1500 and 2500 . As a result, the size of the threshold needs to be carefully selected because a large threshold leads to an increase the probability of misdetections, whereas too small of a threshold may result in an increase in the probability of false detected features.

When comparing FAST-9 and FAST-12 under the same threshold size, it can be observed that FAST-12 detects only the very strong points. Thus, many corner points which are detected by the FAST- 9 are not detected by FAST-12. The probability of the misdetection of features is higher by FAST12 than those by FAST-9. On the other hand, FAST-9 tends to detect more false features (features which do not belong to the crowd) than FAST-12. 

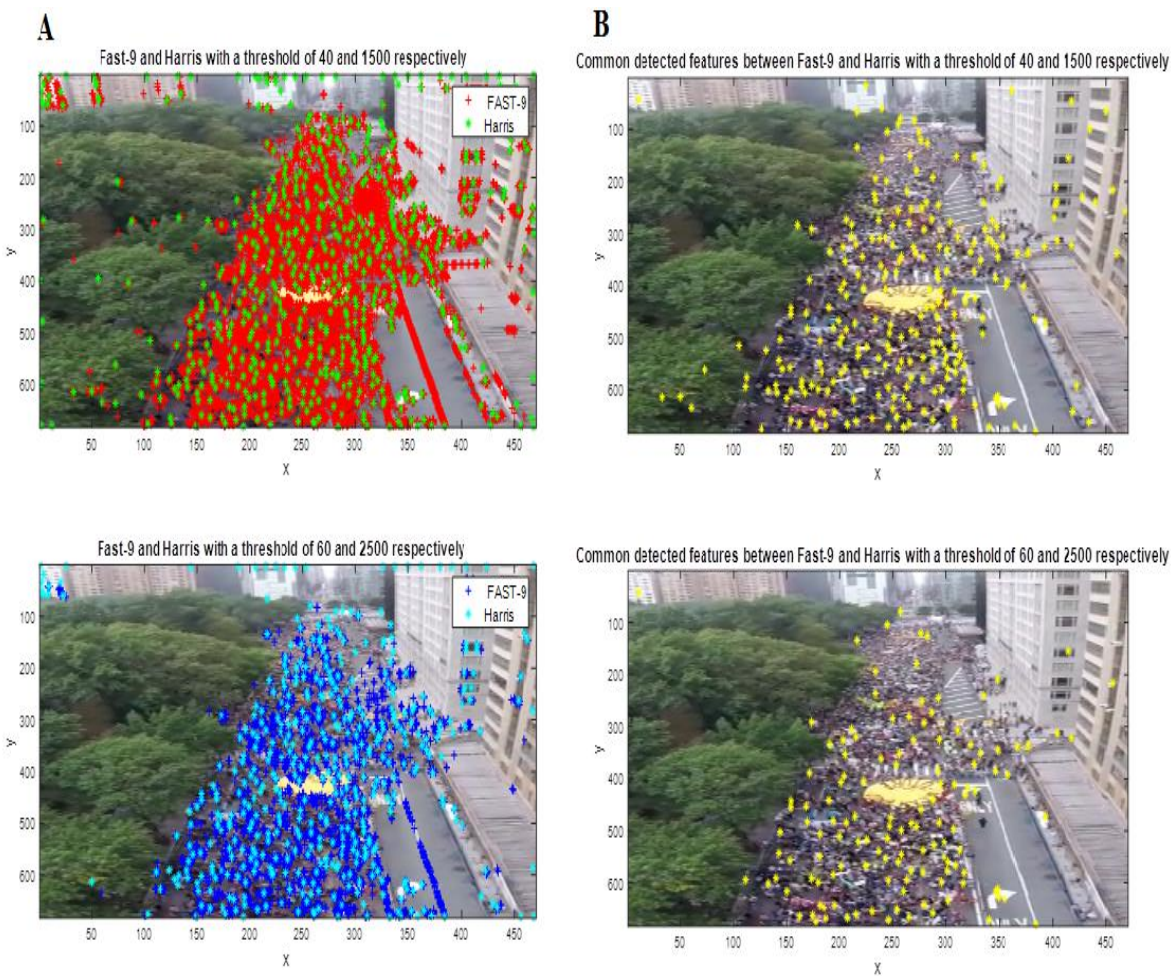

Figure 3. Detection of crowd features based on FAST-9 and Harris (A) and common detected features between FAST-9 and Harris (B) with a threshold of 40 and 60 for FAST-9 and 1500 and 2500 for Harris.

Other comparison between the corner detector methods can be noticed when comparing the common detected features between either FAST-9 and Harris or FAST-12 and Harris. There are more common detected features between FAST-9 and Harris than those between FAST-12 and Harris. The number of common detected features increases as a function of the threshold values. An increase in threshold values leads to an increase the probability of redetected features and vice versa. This situation is presented in Table 2. 
A

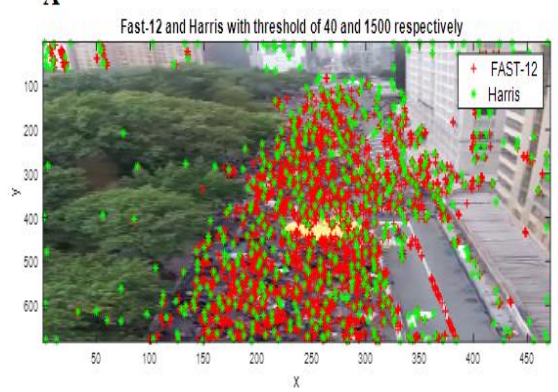

Fast-12 and Harris with threshold of 60 and 2500 respectively

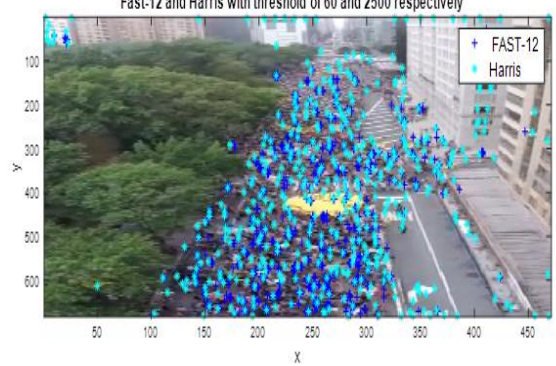

B

Common detected features between Fast-12 and Harris with a threshold of 40 and 1500 respectively

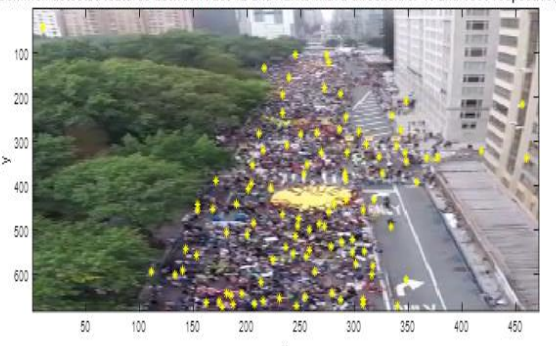

Common detected features between Fast-12 and Harris with a threshold of 60 and 2500 respectively

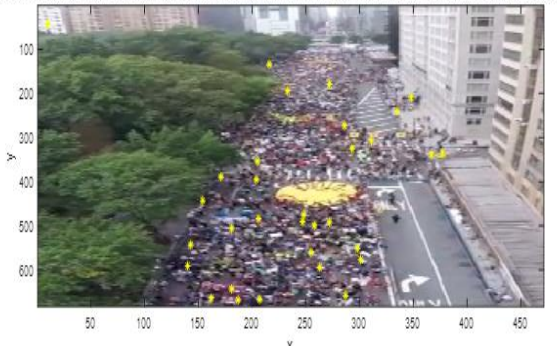

Figure 4. Detection of crowd features based on FAST-12 and Harris (A), and common detected features between FAST-12 and Harris (B) with a threshold of 40 and 60 for FAST-9 and 1500 and 2500 for Harris

Table 2. The number of detected crowd features using the FAST-9, FAST-12 and Harris detectors as a function of the threshold sizes

\begin{tabular}{|c|c|c|c|c|c|c|}
\hline \multirow[b]{2}{*}{$\begin{array}{c}\text { FAST } \\
\text { method } \\
\text { Threshold }\end{array}$} & \multirow{2}{*}{$\begin{array}{c}\text { FAST-9 } \\
\begin{array}{c}\text { Number } \\
\text { of } \\
\text { detected } \\
\text { features }\end{array}\end{array}$} & \multirow{2}{*}{\begin{tabular}{l} 
FAST- \\
\multicolumn{1}{c}{12} \\
Number \\
of \\
detected \\
features
\end{tabular}} & \multicolumn{2}{|c|}{ Harris detector } & \multirow[b]{2}{*}{$\begin{array}{l}\text { The number } \\
\text { of common } \\
\text { detected } \\
\text { features } \\
\text { between the } \\
\text { FAST-9 and } \\
\text { the Harris }\end{array}$} & \multirow{2}{*}{$\begin{array}{c}\text { The } \\
\text { number of } \\
\text { common } \\
\text { detected } \\
\text { features } \\
\text { between } \\
\text { the FAST- } \\
12 \text { and the } \\
\text { Harris }\end{array}$} \\
\hline & & & $\begin{array}{c}\text { Harris } \\
\text { threshold }\end{array}$ & $\begin{array}{c}\text { Number } \\
\text { of } \\
\text { detected } \\
\text { features }\end{array}$ & & \\
\hline 30 & 25552 & 8530 & 1000 & 686 & 335 & 162 \\
\hline 40 & 14004 & 3957 & 1500 & 589 & 247 & 102 \\
\hline 50 & 7626 & 1762 & 2000 & 515 & 179 & 66 \\
\hline 60 & 3893 & 777 & 2500 & 458 & 129 & 32 \\
\hline
\end{tabular}


The performances of all detection methods used herein were evaluated in terms of correctness and completeness (Figure 5). To test the performances of each method, a reference dataset was chosen for comparison purposes. In this scenario, FAST-9 and FAST-12 with a threshold of 40 were chosen as the reference detected points while the Harris detector is the experimental detected points. Then the correctness and completeness of Harris detector was evaluated under four different threshold values. The threshold values for Harris were 1000, 1500, 2000, and 2500 (Table 3).

Table 3. Completeness and correctness of FAST-9 vs Harris, FAST-12 vs Harris detectors as a function of the threshold sizes

\begin{tabular}{|c|c|c|c|c|}
\hline \multirow{2}{*}{$\begin{array}{c}\text { Harris } \\
\text { Thresholds }\end{array}$} & \multicolumn{2}{|c|}{ FAST-9 vs Harris } & \multicolumn{2}{c|}{ FAST-12 vs Harris } \\
\cline { 2 - 5 } & $\begin{array}{c}\text { Completeness } \\
\%\end{array}$ & Correctness \% & Completeness \% & $\begin{array}{c}\text { Correctness } \\
\%\end{array}$ \\
\hline $\mathbf{1 0 0 0}$ & 5 & 50 & 3 & 24 \\
$\mathbf{1 5 0 0}$ & 8 & 40 & 5 & 17 \\
$\mathbf{2 0 0 0}$ & 9 & 35 & 7 & 13 \\
$\mathbf{2 5 0 0}$ & 11 & 28 & 9 & 10 \\
\hline
\end{tabular}

Figure 5 shows the correctness and completeness of the Harris detector as a function of the threshold. The correctness values decrease with an increase of the threshold values. This means that the increase of false positive detected points leads to a decrease the correctness values. For the completeness values however, opposite behavior can be noticed; a decrease in false negative points lead to increase the completeness values. This is also evident when comparing either the FAST-9 or FAST-12 and the Harris detector. 

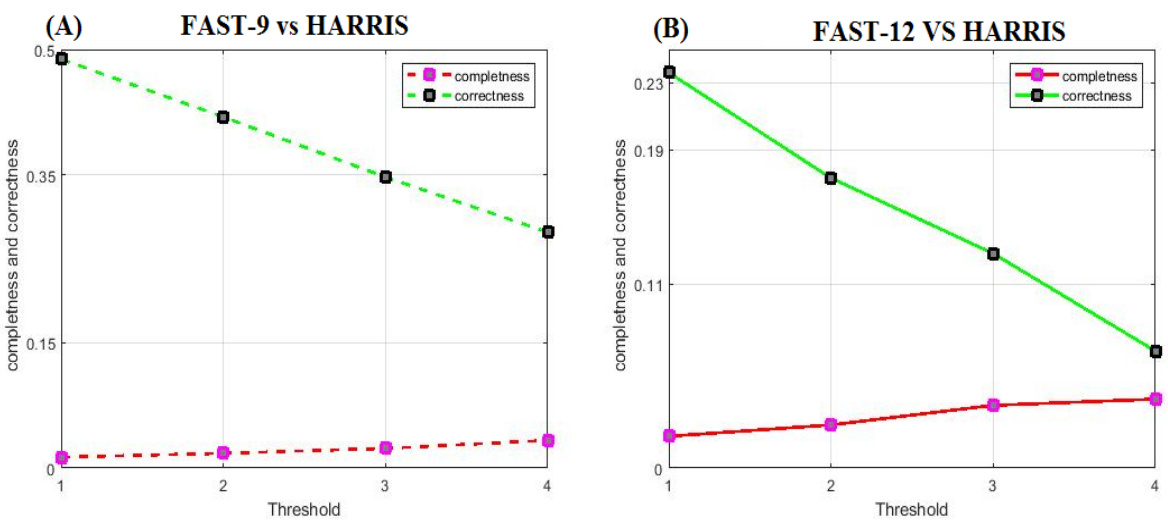

Figure 5. Completeness and correctness for the FAST-9 and the Harris detector (A) and for the FAST-12 and the Harris (B) as a function of threshold values. The threshold numbers are as follows 1,2, 3 and 4 are the Harris thresholds presented in table 3

\subsection{CARS FEATURES EXTRACTION AND QUANTITATIVE EVALUATION}

Figure 6, 7 and 8 present car detection using the FAST-9, FAST-12, the Harris detector and the common detected features (Table 4). The detected cars were extracted using MATLAB codes from UAV images which showed cars in a parking. The detected corners do not reflect the actual number of the cars but instead numerous corner points on the top of each car. This is due to the fact that each car consists of a number of corners which should be detected by the corner detection methods. As identified earlier in this research, FAST-9 detects more points than FAST-12 (Figure 6). In Figure 7, the cars were detected by the Harris detector with a threshold value of 4000 . The Harris detector tends to be more accurate than both the FAST-9 and FAST-12 because very few feature points are detected falsely when compared with FAST-9 and FAST-12. 


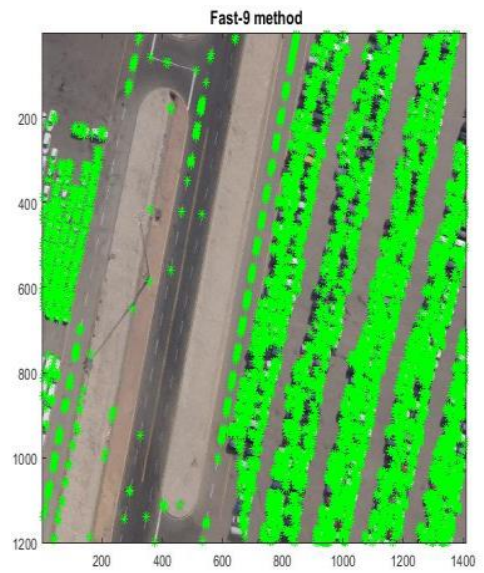

A

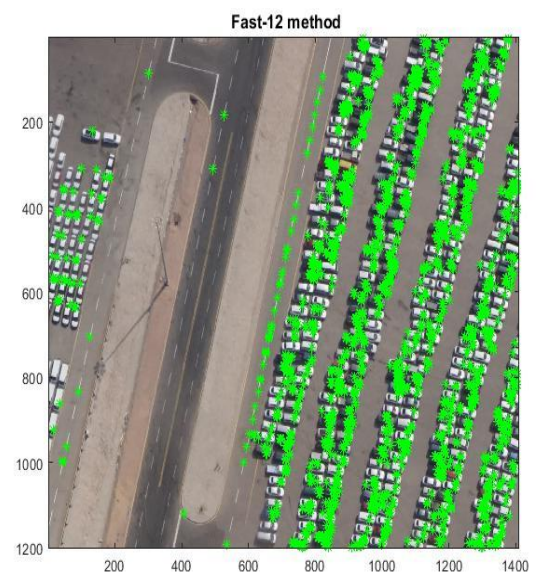

B

Figure 6. Cars detection using FAST-9 (A) and FAST-12 (B) with threshold of 60 .

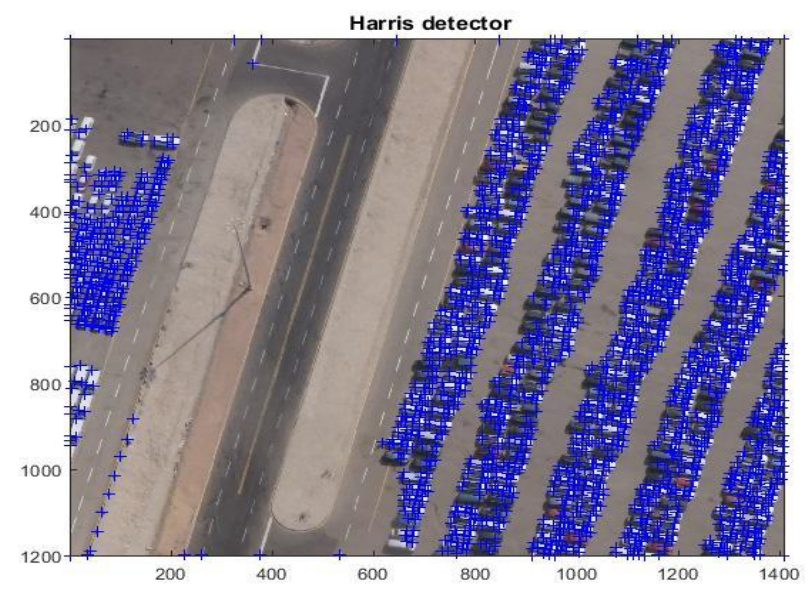

Figure 7. Cars detection using Harris detector with a threshold of 4000

The common detected corners between the Harris and both of the FAST-9 and FAST-12 are presented in Figure 8. The number of common detected corners between FAST-9 and the Harris detector is more than those between 
FAST-12 and the Harris detector. This is due to the fact that FAST-9 has a large number of the detected points which results in an increase in probability of the number of common points between is and the Harris detector.

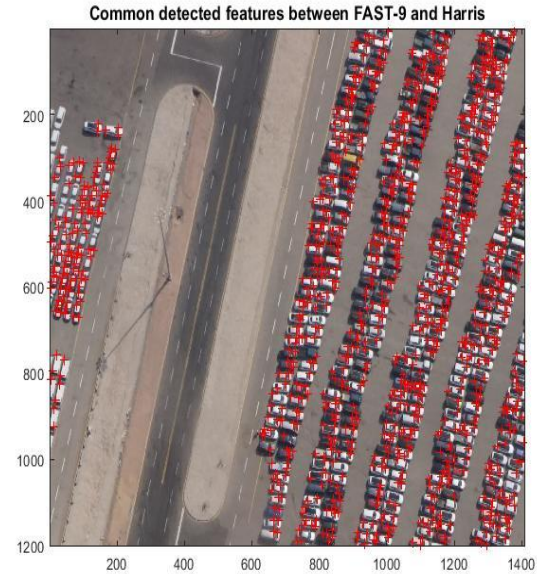

A

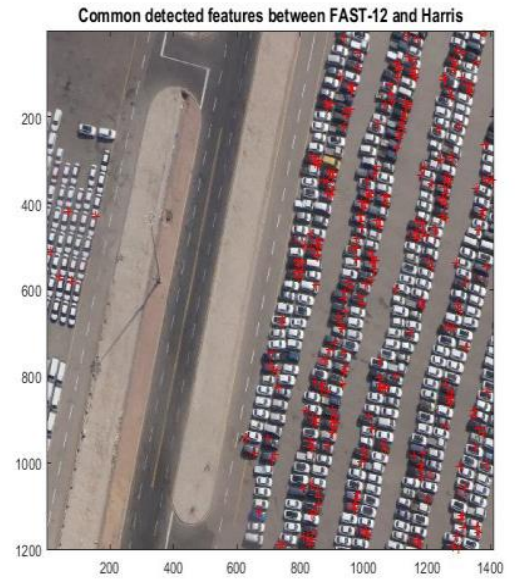

B

Figure 8. Common detected cars between FAST-9 and Harris (A) and FAST-12 and Harris (B)

Table 4. The number of detected crowd features using the FAST-9, FAST-12 and Harris detectors as a function of the threshold sizes

\begin{tabular}{|c|c|c|c|c|c|c|}
\hline \multirow[b]{2}{*}{$\begin{array}{c}\text { FAST } \\
\text { method } \\
\text { Threshold }\end{array}$} & \multirow{2}{*}{$\begin{array}{c}\text { FAST- } \\
\mathbf{9} \\
\text { Number } \\
\text { of } \\
\text { detected } \\
\text { features }\end{array}$} & \multirow{2}{*}{\begin{tabular}{l}
\multicolumn{1}{|c}{ FAST- } \\
$\begin{array}{l}\text { Number } \\
\text { of } \\
\text { detected } \\
\text { features }\end{array}$
\end{tabular}} & \multicolumn{2}{|c|}{ Harris detector } & \multirow{2}{*}{$\begin{array}{l}\text { The number } \\
\text { of common } \\
\text { detected } \\
\text { features } \\
\text { between the } \\
\text { FAST-9 and } \\
\text { the Harris }\end{array}$} & \multirow{2}{*}{$\begin{array}{c}\text { The number of } \\
\text { common } \\
\text { detected } \\
\text { features } \\
\text { between the } \\
\text { FAST-12 and } \\
\text { the Harris }\end{array}$} \\
\hline & & & $\begin{array}{l}\text { Harris } \\
\text { threshold }\end{array}$ & $\begin{array}{l}\text { Number } \\
\text { of } \\
\text { detected } \\
\text { features }\end{array}$ & & \\
\hline 60 & 18951 & 3333 & 4000 & 2312 & 1062 & 274 \\
\hline
\end{tabular}


The performance of the Harris detector was evaluated in terms of correctness and completeness. In this scenario, FAST-9 and FAST-12 were chosen as reference detected points while the feature points detected by Harris detector were the experimental features. As can be seen in Table 5, the completeness is very similar in both cases; however the correctness is 0.57 when using FAST-12 and 0.92 with FAST-9. The threshold value chosen in this detection scenario is 60 and 4000 for both FAST and the Harris respectively. This means that the correctness increases when many false positive points increases and vice versa.

Table 5. Completeness and correctness of detected cars based on the FAST9, FAST-12 and the Harris detector

\begin{tabular}{|l|c|c|c|c|}
\hline Completeness & Correctness & $\begin{array}{c}\text { Common with } \\
\text { Harris }\end{array}$ & $\begin{array}{c}\text { Percentages \% } \\
\text { of common } \\
\text { detected features }\end{array}$ \\
\hline Fast_12 & 0.51 & 0.57 & 683 & 7.5 \\
\hline FAST-9 & 0.50 & 0.92 & 3110 & 5.6 \\
\hline
\end{tabular}

\section{CONCLUSIONS}

Using different threshold sizes, crowd and cars detection was performed to test the impact of threshold values on the performance of each detector method. It has been emphasized that a small threshold value yields many false detected points while a large threshold only detects the strongest feature points (points which are greatly deviated from others). Furthermore, the number of common detected features between FAST-9 and the Harris increases when the threshold values are increased. Therefore, in any corner detector method, selection of a proper size of threshold is a crucial factor for determining the number of detected feature points.

When testing the performance of FAST-9, FAST-12, and Harris detector in terms of completeness and correctness, one detector method was chosen as reference detected points and the other method was the experimental detected points. In this research, when using either the FAST-9 or FAST-12 as the reference detected points and the Harris is the 
experimental detected points, the correctness decreases gradually as a function of threshold values. However, opposite behavior is found in case of completeness as such increase in the threshold value leads to an increase in the completeness.

In the case of cars detection, the Harris detector performs better than the FAST-9 and FAST-12. This has been proven when comparing these methods in terms of the number of false positive and negative detected points. In the Harris method, very few false negative detected points were found when comparing the FAST-9 and FAST-12 methods. It was also found that the percentage of the number of common detected features between the FAST-9 and the Harris in case of cars detection is more than those for crowd detection.

Future research will focus on combining detector methods for objects detection in UAVs images. Furthermore, feature combination will be further investigated to improve the quality and accuracy of the detector methods.

Acknowledgments: The authors would like to express their gratitude to MARS Robotics Company for collecting and providing the data utilized in this research. The authors would also like to thank Yarmouk University for providing the financial support for this research.

Conflicts of Interest: The authors declare no conflict of interest. 


\section{REFERENCES}

Almagbile A. Estimation of crowd density from UAVs images based on corner detection procedures and clustering analysis. Geospatial Information Science. 2019 Jan 2;22(1):23-34.

Biadgie Y, Sohn KA. Feature detector using adaptive accelerated segment test. In2014 International Conference on Information Science \& Applications (ICISA). 2014 May 6; (pp. 1-4). IEEE.

Blaschke, T., and Strobl, J. What's wrong with pixels? Some recent developments interfacing remote sensing and GIS, GeoInformations-Systeme. 2001. Vol. 14, Issue 6, pp. 12-17.

Blaschke T. Object based image analysis for remote sensing. ISPRS journal of photogrammetry and remote sensing. 2010 Jan 1;65(1):2-16.

Blaschke T, Hay GJ, Kelly M, Lang S, Hofmann P, Addink E, Feitosa RQ, Van der Meer F, Van der Werff H, Van Coillie F, Tiede D. Geographic object-based image analysis-towards a new paradigm. ISPRS journal of photogrammetry and remote sensing. 2014 Jan 1;87:180-91.

Burnett C, Blaschke T. A multi-scale segmentation/object relationship modelling methodology for landscape analysis. Ecological modelling. 2003 Oct 15;168(3):233-49.

Davies AC, Yin JH, Velastin SA. Crowd monitoring using image processing. Electronics \& Communication Engineering Journal. 1995 Feb 1;7(1):37-47.

Faille F. Adapting Interest Point Detection to Illumination Conditions. In Digital Image Computing: Techniques and Applications (DICTA). 2003 Dec 10; (pp. 499-508).

Fradi H, Dugelay JL. Crowd density map estimation based on feature tracks. In2013 IEEE 15th International Workshop on 
Multimedia Signal Processing (MMSP). 2013 Sep 30, (pp. 040-045). IEEE.

Harris CG, Stephens M. A combined corner and edge detector. InAlvey vision conference. 1988 Aug 31; (Vol. 15, No. 50, pp. 10-5244).

Hay GJ, Castilla G. Object-based image analysis: strengths, weaknesses, opportunities and threats (SWOT). International Archives of Photogrammetry, Remote sensing, and Spatial Information Sciences OBIA, 2006 Jul 4 (pp. 4-5).

Heipke C, Mayer H, Wiedemann C, Jamet O. Evaluation of automatic road extraction. International Archives of Photogrammetry and Remote Sensing. 1997 Sep;32(3 SECT 4W2):151-60.

Kong D, Gray D, Tao H. A viewpoint invariant approach for crowd counting. In18th International Conference on Pattern Recognition (ICPR'06). 2006 Aug 20 (Vol. 3, pp. 1187-1190). IEEE.

Lambers K, Eisenbeiss H, Sauerbier M, Kupferschmidt D, Gaisecker T, Sotoodeh S, Hanusch T. Combining photogrammetry and laser scanning for the recording and modelling of the Late Intermediate Period site of Pinchango Alto, Palpa, Peru. Journal of archaeological science. 2007 Oct 1;34(10):1702-12.

Ma R, Li L, Huang W, Tian Q. On pixel count based crowd density estimation for visual surveillance. InIEEE Conference on Cybernetics and Intelligent Systems. 2004 Dec 1 (Vol. 1, pp. 170-173). IEEE.

Mair E, Hager GD, Burschka D, Suppa M, Hirzinger G. Adaptive and generic corner detection based on the accelerated segment test. InEuropean conference on Computer vision. 2010 Sep 5; (pp. 183-196). Springer, Berlin, Heidelberg.

Marana AN, Costa LD, Lotufo RA, Velastin SA. On the efficacy of texture analysis for crowd monitoring. InProceedings 
SIBGRAPI'98. International Symposium on Computer

Graphics, Image Processing, and Vision (Cat. No. 98EX237). 1998 Oct 20 (pp. 354-361) IEEE.

Meynberg O, Cui S, Reinartz P. Detection of high-density crowds in aerial images using texture classification. Remote Sensing. 2016 Jun;8(6):470.

Nagai M, Chen T, Shibasaki R, Kumagai H, Ahmed A. UAV-borne 3$\mathrm{D}$ mapping system by multisensor integration. IEEE Transactions on Geoscience and Remote Sensing. 2009 Mar;47(3):701-8.

Patterson, M., and Brescia, A. Integrated sensor systems for UAS, Proceedings of the 23rd Bristol International Unmanned Air Vehicle Systems (UAVS) Conference. 07-09 April 2008, Bristol, United Kingdom

Perko R, Schnabel T, Fritz G, Almer A, Paletta L. Counting people from above: Airborne video based crowd analysis. OAGM/AAPR Workshop. 2013 Apr 23. (arXiv:1304.1876)

Rosten E, Drummond T. Fusing points and lines for high performance tracking. InICCV. 2005 Oct 17, (Vol. 2, pp. 1508-1515).

Rosten E, Porter R, Drummond T. Faster and better: A machine learning approach to corner detection. IEEE transactions on pattern analysis and machine intelligence. 2008 Nov 17;32(1):105-19.

Rottensteiner F, Trinder J, Clode S, Kubik K. Building detection by fusion of airborne laser scanner data and multi-spectral images: Performance evaluation and sensitivity analysis. ISPRS Journal of Photogrammetry and Remote Sensing. 2007 Jun 1;62(2):135-49.

Schmid C, Mohr R, Bauckhage C. Evaluation of interest point detectors. International Journal of computer vision. 2000 Jun 1;37(2):151-72. 
Sirmacek B, Reinartz P. Automatic crowd analysis from very high resolution satellite images. Int. Arch. Photogramm. Remote Sens. Spatial Inf. Sci. 2011 Oct 5;38:3.

Wang B, Bao H, Yang S, Lou H. Crowd Density Estimation Based on Texture Feature Extraction. journal of multimedia. 2013 Aug 1;8(4).

$\mathrm{Xu} \mathrm{C}$, Bao H, Zhang L, He N. Crowd density estimation based on improved Harris \& OPTICS Algorithm. Journal of Computers. 2014 May 1;9(5):1209-17.

Yang, D., Gonzalez-Banos, H., Guibas, L. Counting people in crowds with a real-time network of simple image sensors, In: Proceedings of Ninth IEEE International Conference on Computer Vision. 2003 Oct $13,122-129$.

Yin JH, Velastin SA, Davies AC. Image processing techniques for crowd density estimation using a reference image. InAsian Conference on Computer Vision. 1995 Dec 5, (pp. 489-498). Springer, Berlin, Heidelberg.

Zhan B, Monekosso DN, Remagnino P, Velastin SA, Xu LQ. Crowd analysis: a survey. Machine Vision and Applications. 2008 Oct 1;19(5-6):345-57. 


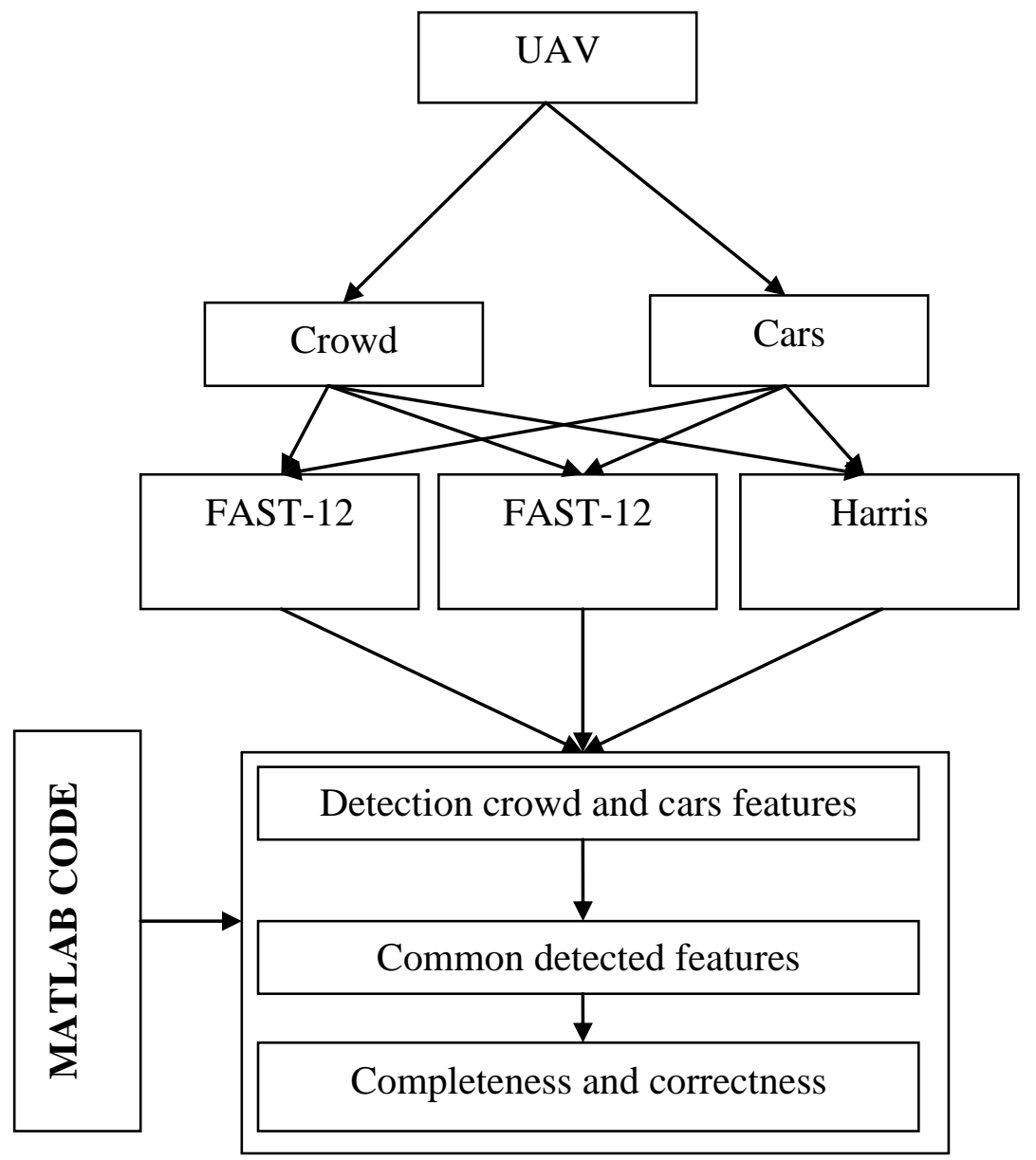

